\title{
Vibration measurement using laser interferometry
}

\section{Guillermo Silva-Pineda, Luis Argote}

Guillermo Silva-Pineda, Luis Ferrer Argote, "Vibration measurement using laser interferometry," Proc. SPIE 3831, Sixth International Conference on Education and Training in Optics and Photonics, (16 June 2000); doi: 10.1117/12.388746 


\title{
Vibration measurement using laser interferometry
}

\author{
Guillermo Silva Pineda ${ }^{1,2}$, Luis Ferrer Argote ${ }^{2,3}$ \\ ${ }^{I}$ Vibrations and Acoustics Division, National Center of Metrology in Mexico, CENAM \\ ${ }^{2}$ Graduate Studies Department of the Engineering Faculty, Autonomous University of Queretaro \\ ${ }^{3}$ D.E.P.F.I. - U.N.A.M.
}

\begin{abstract}
This paper reviews the theoretical basis of two laser interferometry vibration measuring methods of high accuracy, i.e., frequency ratio and Bessel function minimum point. These techniques are used for low and high vibration frequencies, respectively. The most recent experimental developments are highlighted and contributions to the classic models are shown.
\end{abstract}

\section{Summary}

High accuracy laser interferometry measurements, are used to calibrate piezoelectric accelerometers with high temporal and dynamical stability. An electrodynamic exciter is used to produce a linear acceleration level within the frequency range from $50 \mathrm{~Hz}$ to $5 \mathrm{kHz}$. The acceleration level at a constant excitation frequency is calculated using two different measuring methods with which the forced displacement on the accelerometer was obtained. The frequency ratio method (fringe counting method) is applied from $50 \mathrm{~Hz}$ to $800 \mathrm{~Hz}$ and, the Bessel function minimum point method (fringe disappearance method) is applied from $800 \mathrm{~Hz}$ to $5 \mathrm{kHz}$. Some discussions regarding the improvement of experimental issues are shown, based on the mathematical models of each measuring method. The vibration exciter generates a complex rigid body motion, which can be characterized considering both axial and transverse movements to estimate its rocking motion. This can be done looking at the tilting movement described by the moving mirror placed between the accelerometer and the moving platform of the exciter or by a frequency sweep test using a triaxial accelerometer. Since the interferometer and vibration exciter foundations are separated to avoid mechanical coupling, any uncontrolled relative motion between them generates measurement errors. Also, a conceptual model used to estimate the standard accelerometer charge or voltage sensitivity is shown, some aspects regarding the quality and stability of the pickup are emphasized.

Some important aspects about the mathematical models and several experimental recommendations are highlighted. The influence that some imperfections have on the measuring beam of the Michelson interferometer is discussed. Finally, Bessel functions, $J_{n}$, $\mathrm{n}=0,1, \ldots 5$, are shown using a number of digits proportional to the highest accuracy in the determination of the laser wavelength, this increases the resolution with respect to other published values.

\section{Introduction}

In the rigid body mechanics there are six dynamic quantities, i.e., linear displacement, linear velocity, linear acceleration, angle of rotation, angular velocity, and angular acceleration. A vibration exciter produces those dynamic quantities exerting a simple harmonic motion (shm) on the surface under test. Generally, an exciter of electrodynamic type has a moving element with a coil through which passes an alternating flow of electric 
current; the coil is placed in a magnetic field generated by a permanent magnet. The electro-magnetic field generates an acceleration force, called Lorentz acceleration force, which is proportional to the product of the magnetic field and the electric current flow through the coil located on the moving element, as was shown by Maxwell [6].

When the vibration exciter produces an acceleration level, it is transferred to a vibration pickup called accelerometer, generally of the piezoelectric type. Piezoelectric accelerometers have shown the best dynamic characteristics, i.e., relatively high sensitivity and frequency linearity, good stability in time and large frequency and amplitude working ranges below its first frequency of resonance [10]. In case of accelerometers, the calibration factor is the sensitivity amplitude.

In general, the term sensitivity is referred to as the ratio between the output and the input, they can be different physical quantities. In case of the acceleration quantity, the piezoelectric accelerometer output is an electric charge, and the input is an acceleration level exerted upon it.-The acceleration level at the input is in $\mathrm{m} / \mathrm{s}^{2}$, and the electric charge output is in Coulombs, $C$. Therefore, the accelerometer sensitivity has units of $p C / \mathrm{m} / \mathrm{s}^{2}$. However, the accelerometer has a low impedance output which is difficult to be measured by an instrument, so it is necessary a charge amplifier to obtain a high impedance output which can be read by a voltmeter, oscilloscope, signal analyzer or any other voltage measuring instrument.

When a piezoelectric accelerometer is connected to a charge amplifier which sensitivity is in $\mathrm{mV} / \mathrm{pC}$; then the sensitivity of the set has units of their individual sensitivities product, i.e., $m V / m / s^{2}$. Nevertheless, since the charge amplifier sensitivity is not enough stable in the time domain, the common practice is to report the sensitivity of the accelerometer alone. Furthermore, there are accelerometers with a built in amplifier whose output is in ac voltage, the sensitivity of those pickups are expressed in $\mathrm{mV} / \mathrm{m} / \mathrm{s}^{2}$; however, they are not used as standard accelerometers because the built in electronics are not designed to work at high acceleration levels and the probability of damage is high.

The shm produced by the exciter is accurately measured using a laser Michelson interferometer. Measuring the displacement amplitude, $d$, and the excitation frequency, $f$, the acceleration level can be calculated using the equation shown below,

$$
D(t)=d \operatorname{Sin}(2 \pi f t+\phi)
$$

where, $D(t), \quad$ is the displacement in shm at time $t$

$d, \quad$ is the displacement amplitude

$f, \quad$ is the excitation frequency in Hertz

$\phi, \quad$ is the initial phase in radians

Deriving equation (1) twice with respect to time, we find the expression for the instantaneous acceleration, $A(t)$,

$$
A(t)=-d(2 \pi f)^{2} \sin (2 \pi f t+\phi)
$$

where, $\boldsymbol{A}(\boldsymbol{t}), \quad$ is the acceleration in the shm at time $t$

$$
d(2 \pi f)^{2}, \quad \text { is the acceleration amplitude }
$$

The terms $\pm \operatorname{Sin}(2 \pi f t+\phi)$, in equations (1) and (2) are phase related. Phase measurements are beyond the scope of this work. We will be interested only in the measurements of magnitude levels.

It is a good practice to keep harmonic components below $-70 \mathrm{~dB}$ from the full amplitude output at the fundamental frequency in order to assume shm [5]. Using a FFT analyzer 
experimentally does this. It is important to remember that odd harmonic components produce higher distortion than even ones. Another way to filter out the noise components of the signal is to use bandpass filters in order to clean up the signal from harmonic distortion components.

As mentioned above, the acceleration level exerted by the vibration exciter is measured using a laser interferometer of Michelson type, and the output voltage from a conditioner connected to an accelerometer is measured with a high accuracy voltmeter. Since the best experimental accuracy of the excited displacement and frequency is obtained using a laser interferometer [9], then measurement accuracy of the accelerometer is increased when its acceleration measurements are referred to those done with an interferometer. The basic theory of the Michelson interferometer is discussed in the next section.

\section{Michelson interferometer fundamentals}

Albert Abraham Michelson was the first American citizen to win the Nobel Prize in physics, this was in 1907 . Many of the leading physicists of the $19^{\text {th }}$ century supported the ether theory, even though it raised a number of questions. Based on experimental results, Maxwell predicted in 1880 that the movement of the earth through the ether should result in a change in the speed of light. While Maxwell thought that this change was impossible to be detected experimentally, Michelson was confident that it could be observed using an interferometer. This led, in 1881 , to Michelson's famous experiment to demonstrate the 'ether drift'. The null result obtained by Michelson led to the rejection of the concept of the ether and laid the foundations for the special theory of relativity [9].

In 1896, Michelson carried out the first measurement of the length of the Pt-Ir bar that was the international prototype of the metre in terms of the wavelength of the red cadmium radiation. While the idea of the wavelength of a monochromatic source as a natural standard of length had been suggested earlier by Babinet and by Fizeau, it was Michelson's work which demonstrated its feasibility and led, in 1960, to the redefinition of the metre in terms of the wavelength of the orange radiation of ${ }^{86} \mathrm{Kr}$. Michelson made several other contributions and experimental research in high accuracy optical instruments, the spectroscope, an experimental approach to the speed of light, wavelength and frequency of different light sources, among others [1]. Figure 1 shows a Michelson laser interferometer which is used to measure mechanical vibrations, it uses a He-Ne laser source, which has a wavelength, $\lambda$, of approximately $632,815 \mathrm{~nm}$.

A He-Ne laser is used in interferometry because it is monochromatic, collimated, spatially and temporally coherent. The displacement and frequency of the exerted shm upon the measuring surface is measured using a Michelson interferometer; the acceleration level can be obtained using equation (2). Before analyzing the basic theory of the Michelson interferometer, it is necessary to consider the wave nature of light. Light is an electromagnetic phenomenon, described by Maxwell. The theory considers two functions in vector space related to time and describing the electric and magnetic fields. However, in cases like this, it is enough to consider only the electric field. This treatment is used for the case of a limited plane wave and is suitable enough as an approach for the experimental purposes of this work [3]. 
The interferometer measurement can be described as follows: a) a laser light source that is divided in two at a beam splitter, generating the reference and the measuring beams, the wavelength of the laser must be known in advance. b) the reference beam is reflected at the beam splitter and goes forward and backward to a fixed mirror, the elapsed time, or phase delay, is proportional to its optical path. c) at the same time, the measuring beam is transmitted through the beam splitter towards the measuring surface, then it is reflected with a phase change proportional to its position and instantaneous velocity, this phenomenon is well known as the "Doppler effect". d) when the measuring beam goes backward, it is recombined with the reference beam at the beam splitter. e) and the two beams go on to the arm where the photodiode which measures the light intensity, $I$, is located.

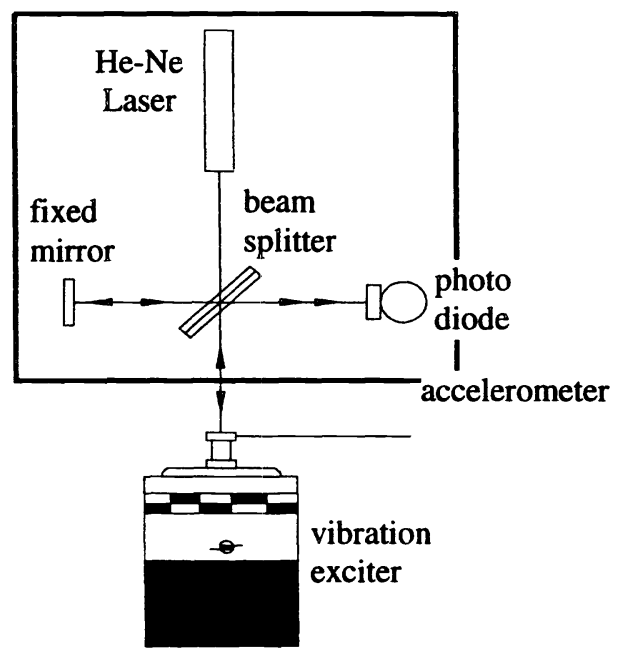

Figure 1. Laser Michelson interferometer used to calibrate accelerometers

The output beam of the laser has an electric field intensity, A, and at the beamsplitter is divided in two identical beams. The beam reflected at the fixed glass mirror is called reference beam, $\boldsymbol{E}_{\boldsymbol{l}}$, and its reflection is phase shifted. The second beam, which is reflected at the measuring surface, is called the measuring beam, $\boldsymbol{E}_{2}$, and its phase it kept constant. The electric field relations for each beam, respectively, and the combined electric field vector when is going towards the beam splitter are shown below,

$$
\begin{aligned}
E_{1} & =A \exp j(\omega t-\varphi) \\
E_{2} & =A \exp j(\omega t+\varphi) \\
E_{1}+E_{2} & =2 A \exp j \omega t \operatorname{Cos} \varphi
\end{aligned}
$$

Both beams are reflected and recombined at the beam splitter producing an interference pattern on the beam that is directed to a fast response photodiode which senses its average intensity, $I$. The light intensity level depends on the phase, $\varphi(t)$, between the beams $E_{1}$ and $\boldsymbol{E}_{2}$. Furthermore, the light intensity level is proportional to the squared sum of the two

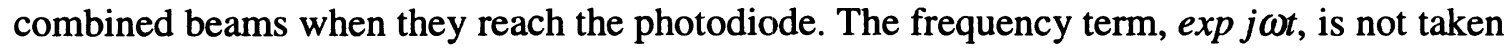
into account because it is not measured with the actual experimental arrangement and is assumed constant during the experiment. So, the light intensity amplitude and the phase can be written in the following form [4], 


$$
I=\left|E_{1}+E_{2}\right|^{2}=A+B \operatorname{Cos} \frac{2 \pi}{\lambda}[\delta+2 \xi(t)]
$$

and

$$
\varphi=\frac{\pi}{\lambda}[\delta+2 \xi(t)]
$$

where, $\lambda$, is the wavelength of the He-Ne laser, $\delta$, is the path difference between the two beams, and the displacement of the shm exerted on the moving mirror or measuring surface is described by the term, $\xi(t)=\xi \operatorname{Sin}(2 \pi f t)$, with an amplitude, $\xi$, and a constant frequency, $f$. Therefore, due to the shm exerted at the measuring surface, the light intensity, $I(t)$, measured by the photodiode varies within a certain range, producing maximum and minimum levels, called interference fringes, shown in figure 2.

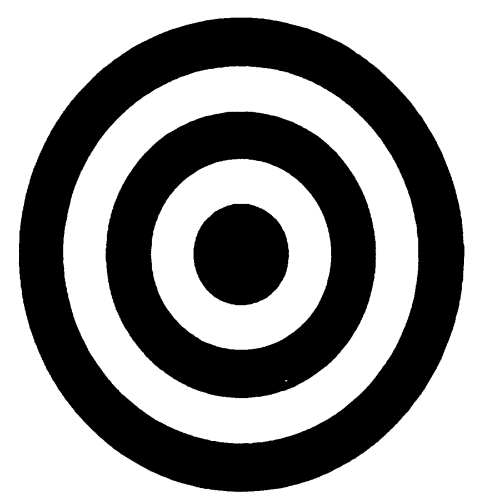

Figure 2. Interference pattern [7]

The equipment used in the experimental arrangement with the Michelson interferometer is shown in figure 3 . On the moving table of the vibration exciter, which is describing a shm, is mounted an accelerometer. The electric output of the accelerometer is proportional to the acceleration level, however, this acceleration level is measured using a laser Michelson interferometer. In this way, the calibration of the accelerometer is improved when it is referred to the acceleration level found using the interferometer.

With the equipment shown in figure 3 and, due to practical limitations of the vibration exciter, the frequency range is from $50 \mathrm{~Hz}$ to $5 \mathrm{kHz}$. Below $50 \mathrm{~Hz}$ the distortion of the exerted movement increases and it is not possible to assume shm, and above $5 \mathrm{kHz}$ appears elastic motion between some parts of the exciter, so above that frequency different acceleration levels are found at different points. There are two possible experimental methods to obtain the acceleration level using the interferometer, i.e., frequency ratio method (fringe counting method) and Bessel function minimum point method (fringe disappearance method). The fringe counting method is used within the frequency range from $50 \mathrm{~Hz}$ to $800 \mathrm{~Hz}$, because the displacement applied is large enough to have a number of fringes suitable to have high accuracy. The higher the frequency, the smaller the displacement and fewer the number of fringes, and the measurement error is increased [10]. 


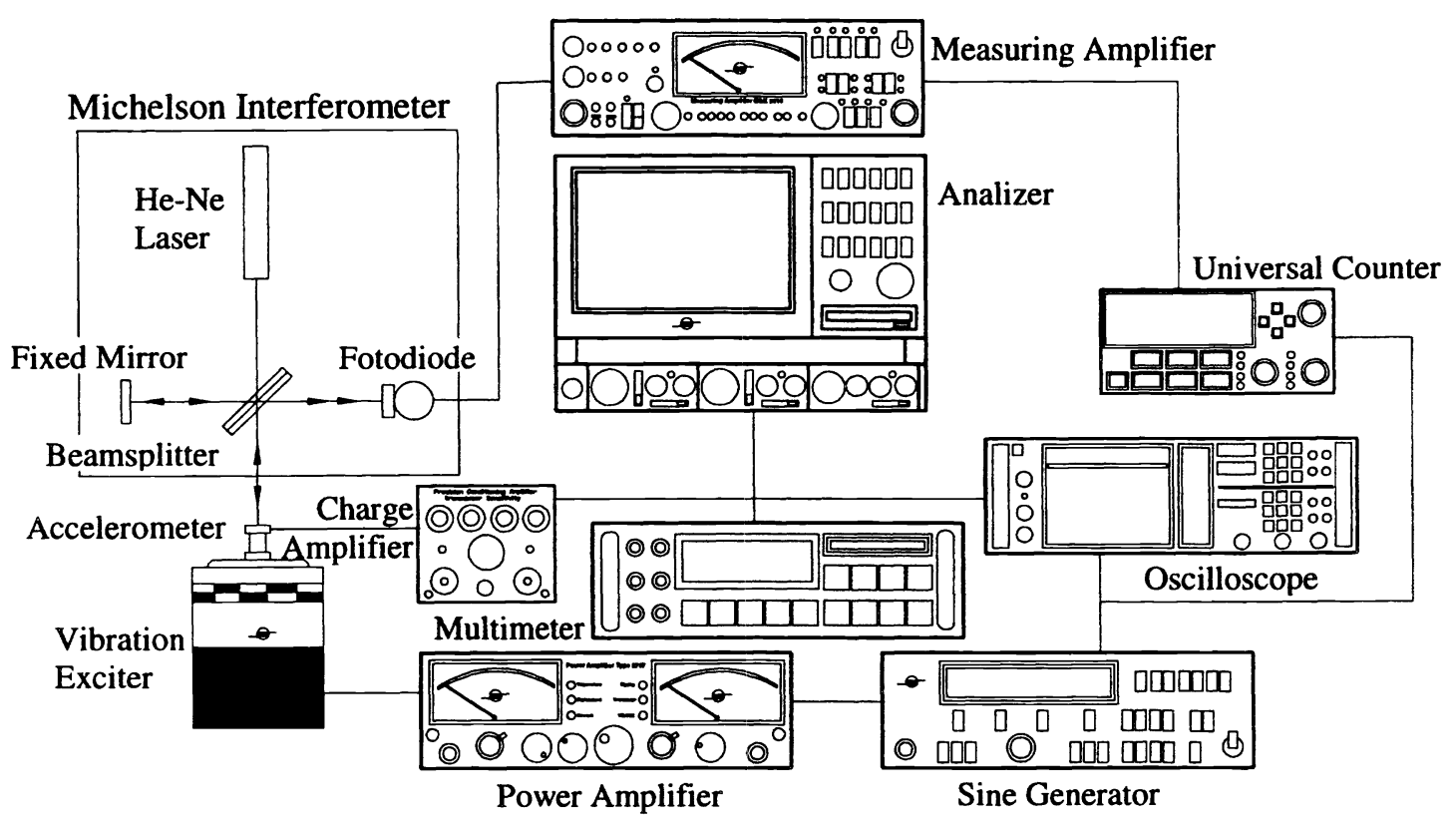

Figure 3. Equipment and experimental arrangement for the Michelson interferometer

At frequencies above $800 \mathrm{~Hz}$, the Bessel function minimum-point method is used. In this method it is necessary to estimate the Power Spectral Density (PSD) of the photodiode output signal, this can be done experimentally using a spectrum analyzer. At a certain frequency and acceleration level, observing the signal spectrum it can be found the minimum points at rertain frequencies which are integer multiples of the excitation frequency. In the next sections the interferometric measuring methods will be described in more detail.

\subsection{Frequency ratio method (fringe counting method)}

This method consists of measuring two different frequencies that are directly related to the dynamic displacement, $\xi(\mathrm{t})$, exerted upon the accelerometer during its calibration. The first of these frequencies is the "excitation frequency" at the vibration exciter that comes from a sine generator. The moving element of the vibration exciter has a metallic plate with a polished surface, the accelerometer is bolted directly over the polished surface. Therefore, the sensitive surface of the accelerometer is in contact with the polished surface, this surface is called the "measuring surface". When the harmonic excitation is applied at the measuring surface, the accelerometer output is referred to both the excitation frequency and the displacement measured with the interferometer [4].

When the measuring surface moves backward or forward a distance of $\lambda / 2$, the fringes move from the dark side to the bright one. So, at a stated frequency and acceleration level, there will be a constant number of fringes, or optical pulses, during each mechanical vibration cycle, in other words, the fringe frequency equals the mechanical frequency times the number of fringes, as shown in figure 4 . This number of fringes is proportional to both the displacement and acceleration levels. 
It was stated in equation ( $5 \mathrm{a}$ and $\mathrm{b}$ ) that the light intensity measured by the photodiode changes in proportion to the forced $s h m$ described by the measuring surface. The light intensity, which indeed is an average value, varies harmonically too. Since the values of the cosine vary in the range from -1 to 1 , we will have the maximum level of light intensity when,

$$
\operatorname{Cos}\left\{\frac{2 \pi}{\lambda}[\delta+2 \xi(t)]\right\} \approx \pm 1
$$

that is to say,

$$
\frac{2 \pi}{\lambda}[\delta+2 \xi(t)]=2 \pi n
$$

where, $n$, is an integer. Considering two consecutive values of the integer, i.e., $n$ and $n+1$, when two consecutive maximums occur. Then, we will find the relationship between the displacements, $\xi_{\mathrm{n}}$ and $\xi_{\mathrm{n}+1}$, respectively. The above means that two consecutive maximums of light intensity are produced when the traveled distance of the measuring surface follows the next relationships,

$$
\frac{2 \pi}{\lambda}[\delta+2 \xi(t)]=2 \pi n
$$

and

$$
\frac{2 \pi}{\lambda}[\delta+2 \xi(t)]=2 \pi(n+1)
$$

From the two equations (7) and (8), the traveled distance of the measuring surface to produce two consecutive light intensity maximums, $\left(\xi_{n+1}-\xi_{n}\right)=\lambda / 2$, can be obtained. When the forced shm at the measuring surface has both constant frequency and constant displacement amplitude, the number of fringes or light maximums is constant too, as shown in figure 4.

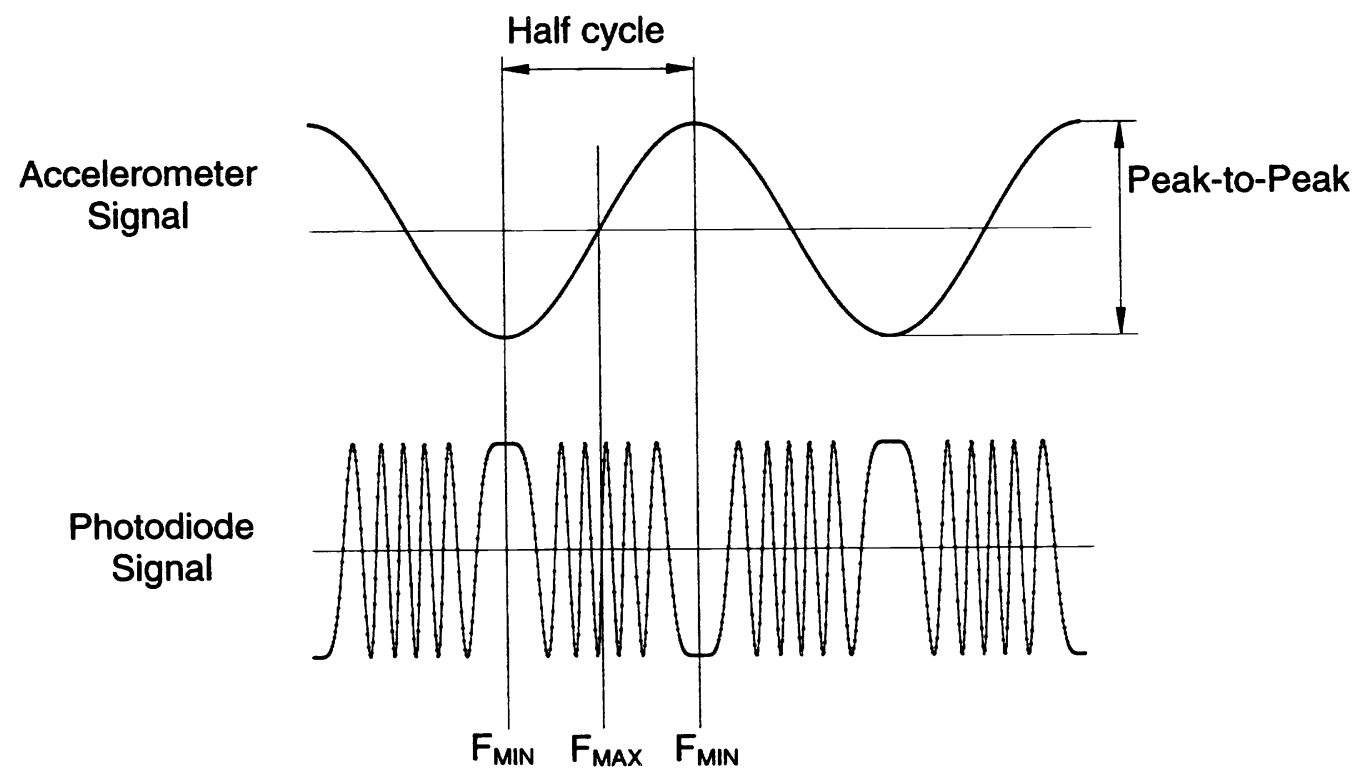

Figure 4. Records of the interferometry fringes and accelerometer signal output 
From the discussion above, we already know that in order to determine the number of fringes one must calculate the dynamic displacement amplitude, $\xi$, of the measuring surface; using the following relation [5],

$$
R_{F}=\frac{\xi \times 2(\text { forward }- \text { backward }) \times 2(\text { peak }- \text { peak }) \times 2(\text { completecycle })}{\lambda}=\frac{\xi 8}{\lambda}
$$

and

$$
R_{F}=\frac{\text { Fringe Frequency }}{\text { shm Frequency }}
$$

or

$$
\xi=\frac{\lambda R_{F}}{8}
$$

where, $R_{F}$, is the number of averaged fringes during several cycles of the $s m h$ described by the measuring surface. It is important to say that for a He-Ne laser, the average wavelength, $\lambda$, is 632,815 nanometers approximately.

This specific experimental implementation shown in figure 3, has several sources of experimental errors, some of which are listed below,

- the shm of the measuring surface has tilting and rocking motion generated by the vibration exciter suspension; in order to avoid this effect it is recommended to use an air bearing suspension,

- since the measuring surface is a metallic mirror, its reflected beam shows speckle, which generates small phase changes in the photodiode measurement [2]. Furthermore, at some points, it shows diffraction due to scratches generated during the manufacturing process, and

- it is recommended that only the laser light reaches the photodiode, otherwise, the noise level of the measurements will be increased [8].

\subsection{Bessel function minimum point method}

When the photodiode output signal, shown in figure 4 , is displayed in the Frequency domain, generates a number of harmonic components which have a frequency spacing between each other equal to the excitation frequency applied to the measuring surface. Experimentally, in order to observe the frequency domain, we must use a spectrum analyzer with a FFT option. To identify every one of the harmonic components displayed in the frequency domain, we must analyze the equations (5a and $5 b$ ). In order to expand the expression of the average light intensity, $I(t)$, we will use the geometric identity shown in equation (11),

$$
\cos (A+B)=\cos A \cos B-\operatorname{sen} A \operatorname{sen} B
$$

then, we must remember the Jacobi's series,

$$
\begin{gathered}
\cos (A \operatorname{sen} B)=J_{0}(A)+2 J_{2}(A) \cos 2 B+2 J_{4}(A) \cos 4 B+\cdots \\
\operatorname{sen}(A \operatorname{sen} B)=2 J_{1}(A) \cos B+2 J_{3}(A) \cos 3 B+\cdots
\end{gathered}
$$

Using the above geometrical identity (equation (11)) and the Jacobi's series (equations (12) and (13)), we find the expanded expression for the average light intensity, $I(t)$, 


$$
\begin{aligned}
I(t)=A & +B \cos \left\{\left(\frac{4 \pi \delta}{\lambda}\right)\left[J_{0}\left(\frac{4 \pi \xi}{\lambda}\right)+2 J_{2}\left(\frac{4 \pi \xi}{\lambda}\right) \cos 2(2 \pi f t)+\cdots\right]\right\} \\
& -B \operatorname{sen}\left\{\left(\frac{4 \pi \delta}{\lambda^{*}}\right)\left[J_{1}\left(\frac{4 \pi \xi}{\lambda}\right) \cos (2 \pi f t)+2 J_{3}\left(\frac{4 \pi \xi}{\lambda}\right) \cos 3(2 \pi f t)+\cdots\right]\right\}
\end{aligned}
$$

In order to determine certain Bessel function minimum points, $J_{n}$, where $\mathrm{n}=0,1, \ldots, 5$; we must observe the frequency domain. When a FFT analyzer displays the power spectral density, $P S D$, of the light intensity, it shows several frequency components. The first frequency component is displayed at the DC component, the second component is displayed at the excitation frequency, and the other frequency components are displayed at harmonics of the excitation frequency applied at the measuring surface. The magnitude of every one of these components depends on the displacement magnitude of the measuring surface, $\xi$. This means that, keeping constant the excitation frequency, the displacement magnitude of the measuring surface, $\xi$, increases and at the displacements stated in the table 1, the PSD will show a minimum at the frequency which corresponds to one Bessel function, $J_{n}$.

\begin{tabular}{|c|c|c|c|c|c|c|}
\hline & $\mathrm{J}_{0}\left(\frac{4 \pi \xi}{\lambda}\right)$ & $J_{1}\left(\frac{4 \pi \xi}{\lambda}\right)$ & $\mathrm{J}_{2}\left(\frac{4 \pi \xi}{\lambda}\right)$ & $J_{3}\left(\frac{4 \pi \xi}{\lambda}\right)$ & $J_{4}\left(\frac{4 \pi \xi}{\lambda}\right)$ & $J_{5}\left(\frac{4 \pi \xi}{\lambda}\right)$ \\
\hline & $\xi[\mathrm{nm}]$ & $\xi[\mathrm{nm}]$ & $\xi[\mathrm{nm}]$ & $\xi[\mathrm{nm}]$ & $\xi[\mathrm{nm}]$ & $\xi[\mathrm{nm}]$ \\
\hline \multirow[t]{2}{*}{0} & 1 & 0 & 0 & 0 & 0 & 0 \\
\hline & 0 & 0 & 0 & 0 & 0 & 0 \\
\hline \multirow[t]{2}{*}{1} & 2,404825 & 3,831705 & 5,135623 & 6,380161 & 7,588343 & 8,771483 \\
\hline & 121,102 & 192,956 & 258,619 & 321,291 & 382,132 & 441,713 \\
\hline \multirow[t]{2}{*}{2} & 5,520079 & 7,015587 & 8,417245 & 9,761023 & 11,064709 & 12,338605 \\
\hline & 277,979 & 353,290 & 423,874 & 491,544 & 557,195 & 621,345 \\
\hline \multirow[t]{2}{*}{3} & 8,653727 & 10,173469 & 11,619841 & 13,015201 & 14,372537 & 15,700175 \\
\hline & 435,783 & 512,314 & 585,150 & 655,417 & 723,770 & 790,627 \\
\hline \multirow[t]{2}{*}{4} & 11,791535 & 13,323691 & 14,795951 & 16,223467 & 17,615967 & 18,980133 \\
\hline & 593,796 & 670,952 & 745,092 & 816,978 & 887,102 & 955,798 \\
\hline \multirow[t]{2}{*}{5} & 14,930917 & 16,470631 & 17,959819 & 19,409415 & 20,826935 & 22,217799 \\
\hline & 751,888 & 829,425 & 904,417 & 977,416 & 1048,799 & 1118,840 \\
\hline
\end{tabular}

Table 1. Bessel function minimum points, $J_{n}, \mathrm{n}=0,1,2,3,4$ and 5

As the laser wavelength is $632,815 \mathrm{~nm}$ approximately, the table 1 states amplitude displacements with bigger resolution than those shown in another references $[5,10]$ but using the same resolution than the laser wavelength, in picometers. During the experimental measurements used in this method it is important to keep low the relative non-controlled displacements between the interferometer and the vibration exciter. It is possible that at frequencies higher that $800 \mathrm{~Hz}$, rocking motion and distortion appears in the forced motion of the exciter, it is important to keep as low as possible these sources of error. It is important to take into-account the sources of error listed below,

- The interferometer alignment is very important to reach the highest measurement accuracy [2]. One must assure that only one fringe is on the sensitive surface of the photodiode during the measurement, 
- The relative uncontrolled motion between the foundation of the vibration exciter and the interferometer is an important source of error [10], therefore it must be kept as low as possible and the mechanical coupling must be avoided too.

- At frequencies higher than $800 \mathrm{~Hz}$, distortion of the shm exerted by the vibration exciter can reduce the experimental accuracy if it shows resonances within the working range [5].

\section{Conclusions}

As Michelson and many other men of science had made, it is very important to be confident that experimental research can guide to new technological and scientific discoveries. To improve the quality of the shm generated by the vibration exciter it should has an air bearing suspension. The accelerometer calibration is traceable to time when upon it is applied constant excitation frequency and its displacement is measured with the interferometer using as a reference the wavelength of the laser. In the fringe counting method, the fringe frequency equals the mechanical excitation frequency times the number of fringes, and the number of fringes is proportional to both the displacement and acceleration levels.

Some factors to be taken into account to get the best accuracy of the fringe counting method are: a) when a metallic mirror is used as a measuring surface, its reflected beam shows speckle and small phase changes due to the surface roughness and flatness; $b$ ) if the measuring surface has tilting and rocking motion, then this affects to the fringe frequency stability and the measurement accuracy is reduced; c) Only the laser light should reach the photodiode in order to reduce the noise level of its output.

For the fringe disappearance method, it is necessary to observe the Power Spectral Density, $P S D$, of the photodiode output using a spectrum analyzer, which shows several frequency components. The first frequency component is displayed at the DC component, the second component is displayed at the excitation frequency, and the other frequency components are displayed at harmonics of the excitation frequency applied at the measuring surface. As the laser wavelength is $632,815 \mathrm{~nm}$ approximately, the displacement amplitude for each disappearance point should be shown, at least, using the same resolution than the laser wavelength, in picometers.

In the fringe disappearance method, the interferometer alignment is very important and only one fringe must be at a time on the sensitive surface of the photodiode during the measurement. The relative uncontrolled motion between the foundation of the vibration exciter and the interferometer is an important source of error, therefore it must be kept as low as possible and the mechanical coupling must be avoided too. At frequencies higher than $800 \mathrm{~Hz}$, distortion of the shm exerted by the vibration exciter can reduce the experimental accuracy if it shows resonances within the working range.

Some research is in progress to improve the measurement accuracy for the interferometer, i.e., the relation between the number of fringes, which impinge at a time the photodiode and the fringe frequency; the measurement error due to a phase shift produced by the roughness and flatness of the surface when a metallic mirror is used as a measuring surface and; errors in the fringe frequency measurement due to tilting and rocking motion of the measuring surface. 


\section{References}

1. A. A. Michelson; Light waves and their uses; The University of Chicago Press, 1903.

2. A. E. Lowman, J. E. Greifenkamp; Interferometer Errors Due to the Presence of Fringes; Engineering \& Laboratory Notes, Optical Society of America, 1996.

3. H.J. Pain; The Physics of Vibrations and Waves; $4^{\text {th }}$ Edition, John Wiley \& Sons, 1997. ISBN 0741937428.

4. H.-J. v. Martens, A. Täubner; Zur theorie interferometrischer schwingungsmessungen; Metrologische Abhandlungen 3, 1983.

5. ISO 5347-1. Methods for the calibration of vibration and shock pick-ups - Primary vibration calibration by laser interferometry.

6. J. C. Maxwell; A treatise on electricity \& magnetism; Dover Publications Inc., New York, 1954.

7. R. Guenther; Modern Optics; John Wiley \& Sons, 1990. ISBN 0471512885.

8. P. Hariharan; Achromatic Phase-Shifting for White-Light Interferometry; Engineering \& Laboratory Notes, Optical Society of America, 1996.

9. P. Hariharan; Optical Interferometry; Academic Press, 1985.

10. P. Hohmann; Untersuchung über die Erhöhung der Auflösung, über die Phasenmessung und über den Einflu $\beta$ von Klirrfaktoren und starken Schallfeldern bei der interferometrichen Messung von Schwingungsamplituden; Dissertation, 1971, TU Braunschweig, Deutschland. 